\title{
Correction to: A copula-based Markov chain model for serially dependent event times with a dependent terminal event
}

\author{
Xin-Wei Huang ${ }^{1} \cdot$ Weijing Wang $^{1} \cdot$ Takeshi Emura $^{2}$ \\ Published online: 15 January 2021 \\ (C) Japanese Federation of Statistical Science Associations 2021
}

\section{Correction to: Japanese Journal of Statistics and Data Science https://doi.org/10.1007/s42081-020-00087-8}

There are transcription errors in the Supplementary Material that were caused by production process.

The correct files and links are given below. The original article has been corrected.

Publisher's Note Springer Nature remains neutral with regard to jurisdictional claims in published maps and institutional affiliations.

The original article can be found online at https://doi.org/10.1007/s42081-020-00087-8.

Electronic supplementary material The online version of this article (https://doi.org/10.1007/s4208 1-020-00099-4) contains supplementary material, which is available to authorized users.

Takeshi Emura

takeshiemura@gmail.com

Xin-Wei Huang

xinweihuang@hotmail.com

Weijing Wang

wjwang@stat.nctu.edu.tw

1 Institute of Statistics, National Chiao Tung University, Hsinchu, Taiwan

2 Department of Information Management, Chang Gung University, No. 259, Wenhua 1st Rd., Guishan Dist., Taoyuan City 333, Taiwan 